\title{
SPECIAL POSITION FOR THE ANAESTHETIC MANAGEMENT OF A PATIENT WITH GIANT NECK AND BACK MASSES
}

\section{AKHLAGHI, G. SHABANIAN and M. ABEDINZADEH}

\author{
Anaesthesiology Department, Shahrekord University of Medical Sciences, Shahrekord, IRAN
}

Corresponding Author: Dr Mahmood Akhlaghi

Conflict of interest:

\section{SUMMARY}

A neck mass is a risk factor for difficult airway during induction of anaesthesia particularly when accompanied with a giant back mass which limits positioning of the patient in the supine position. We report the management of a young woman with known recurrent neurofibroma scheduled for resection of her giant neck and back masses. Unfortunately, her dorsal thoracic mass was too huge to allow adoption of the supine position for induction of anaesthesia. In order to achieve a suitable supine position which is fundamental during airway management, we created a hole in the operating table to fit her giant back mass. Exhibition of this special operating table may help to achieve a suitable position and ease airway management which is not possible with routine tables in cases with giant posterior masses.

Keywords: Anaesthetic position, Operating table, Giant mass, Airway management, General anaesthesia

\section{INTRODUCTION}

A huge neck mass is a risk factor for difficult airway during induction of anaesthesia and this has been reported in some studies ${ }^{1-3}$ Together with giant thoracic masses the difficult airway is more compounded particularly when the mass is too huge to allow the patient to be placed in the supine position. We report a case of recurrent neurofibroma with multiple huge masses in her neck and back which needed special attention before induction of anaesthesia.

\section{CASE REPORT}

A 36 year old female patient with known recurrent neurofibroma was scheduled for reoperation. She complained of dyspnoea and had a mild acidosis. Her arterial blood gases $(\mathrm{ABG})$ were as follows $\mathrm{PH}=7.33 \mathrm{~Pa}-$ $\mathrm{Co} 2=35 \mathrm{HCo} 3=18.9$. She was fully alert but her oxygen saturation (SpO2) was low $(87 \%)$ without any oxygen supplementation. Clinically the patient had multiple huge masses in her neck and back and the latter was so huge that adoption of the supine position was limited. Huge masses as well as significant tracheal devia-

\section{E-mail: akhlaghi236@yahoo.com}

tion were revealed in spiral computed tomography scans (Figure 1).

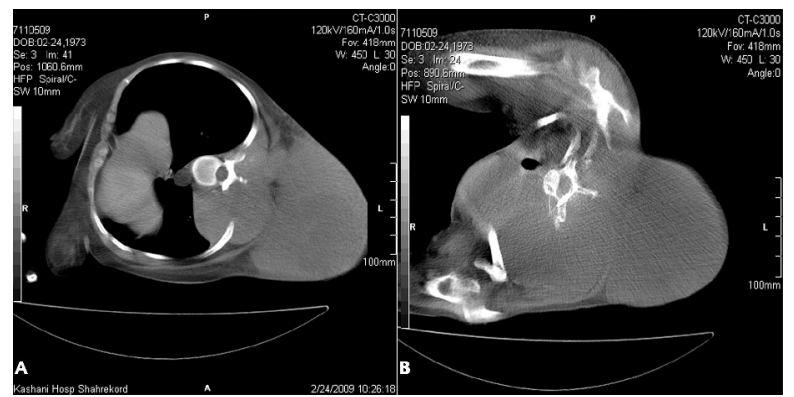

Figure 1 Spiral Computed Tomography shows: A) A giant dorsal mass, B) Teacheal deviation due to a huge neck mass

This made the induction of anaesthesia using the conventional theatre table impossible and therefore the table was modified to create a big hole to accommodate the patient's huge dorsal mass (Figures 2and 3).

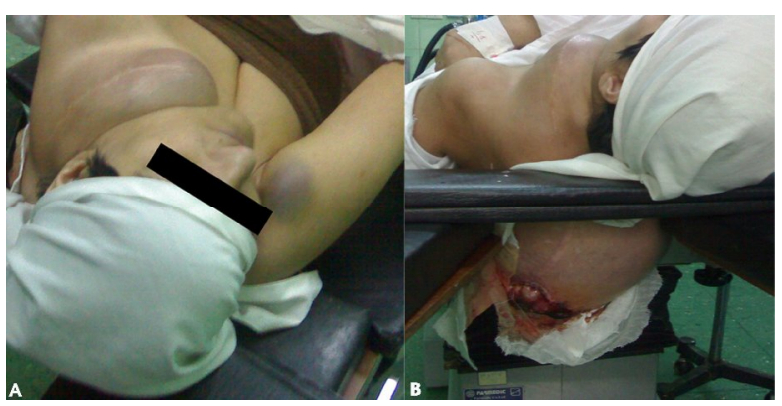

Figure 2 Special position for patient with multiple huge masses. A) Huge neck mass, B) Giant back mass with necrotic tip fitted in the operating table

The modification of the table allowed the supine positioning of the patient. Blind nasotracheal intubation was performed after pre-oxygenation and sedating the patient with midazolam $2 \mathrm{mg}$ and fentanyl $150 \mu \mathrm{g}$ intravenously as well as lubrication with lignocaine $2 \%$ jelly. Induction of anaesthesia was performed using intravenous sodium thiopental $5 \mathrm{mg} / \mathrm{kg}$ plus atracurium 
$0.5 \mathrm{mg} / \mathrm{kg}$ followed by infusing propofol $(120 \mu \mathrm{g} / \mathrm{kg} / \mathrm{min})$ for maintenance of anaesthesia. Thereafter, surgery was done and the masses were successfully removed. The patient was discharged a few days later for further follow up.

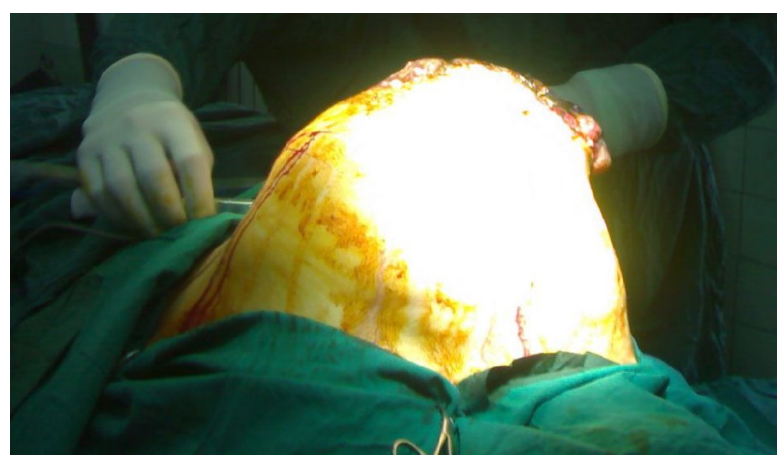

Figure 3 Patient with giant back mass in prone position

\section{DISCUSSION}

Airway management of patients with huge neck masses is a challenge for anaesthetists. Preoperative assessment of such cases particularly imaging studies may indicate the ease of intubation. It is important to maintain spontaneous ventilation until after successful intubation. Apart from direct risk factors, other indirect risk factors to difficult intubation like positioning need to be recognised. Therefore, an appropriate position must be considered before laryngoscopy. Failure to recognise this would lead to major airway maintenance difficulties for the anaesthetic team. Along with the huge neck and back masses, there was also a marked tracheal deviation that created more problems before induction of anaesthesia with compression on trachea, our case had a giant back mass made more challenge for us before induction of anaesthesia.

Positioning of the patient in the appropriate position could reduce complications during surgery. ${ }^{4,5}$ To our knowledge, there is no special operating table to facilitate a suitable position for these particular patients. During the last decade, different models of operating tables have been exhibited. ${ }^{6-8}$ None of these tables nor a standard one could facilitate the proper positioning of this patient because of the huge dorsal mass. A hole was created in the upper part of the operating table to allow the patient to be placed in an optimal supine position to manage the airway as well as facilitate the successful removal of the neck mass. Fortunately, no complications were observed during and after the longperiod of anaesthesia and surgery.

\section{CONCLUSION}

Proper positioning is very important for induction of anaesthesia and securing the airway and this is best done in supine position. Huge back masses interfere with a suitable supine position and may be a risk factor for difficult airway particularly when it coexists with a huge neck mass. Exhibition of a special modified operating table may help to ease airway management and reduce complications related to positioning.

\section{REFERENCES}

1. Dabbagh A, Mobasseri N, Elyasi H, Gharaei B, Fathololumi M, Ghasemi M, et al. A rapidly enlarging neck mass: the role of the sitting position in fiberoptic bronchoscopy for difficult intubation. Anesth Analg 2008 Nov;107(5):1627-9.

2. Huda A, Hamid M. Airway management in a patient with huge neck mass. J Pak Med Assoc 2008 Oct;58(10):574-5.

3. Leva E, Pansini L, Fava G, Maestri L, Pansini A, Selvaggio $\mathrm{G}$. The role of the surgeon in the case of a giant neck mass in the EXIT procedure $J P e$ diatr Surg 2005 Apr;40(4):748-50.

4. Papadonikolakis A, Wiesler ER, Olympio MA, Poehling GG. Avoiding catastrophic complications of stroke and death related to shoulder surgery in the sitting position. Arthroscopy 2008 Apr;24(4):481-2.

5. Ralston C, Tatman A. An audit of serious complications during neurosurgery in children using the sitting position Paediatr Anaesth 2000;10(6):6989.

6. Arai S, Yoshioka K, Suzuki C, Takahashi H, Itoh T, Nakano S. Development of a neurosurgical operating table for adult cattle and changes in intracranial pressure and blood pressure in adult cattle undergoing long-time isoflurane anesthesia. J Vet Med Sci 2006 Apr;68(4):337-43.

7. Eipe N., Mahajan K. A self-contained operating table Anaesthesia 2007; 62(3): 294.

8. Matthiews J. New Eschmann operating table. British Journal of Perioperative Nursing 2002;12(2):59. 